\title{
Switching current distributions and macroscopic quantum tunneling in over-doped BSCCO mesas with nanometer thickness
}

\section{$\operatorname{AUTHOR(S):~}$}

Kakeya, Itsuhiro; Hamada, K; Tachiki, T; Watanabe, T; Suzuki, M

\section{CITATION:}

Kakeya, Itsuhiro ...[et al]. Switching current distributions and macroscopic quantum tunneling in over-doped BSCCO mesas with nanometer thickness. Superconductor Science and Technology 2009, 22(11): 114014.

\section{ISSUE DATE:}

2009-11-01

URL:

http://hdl.handle.net/2433/123405

\section{RIGHT:}

This is an author-created, un-copyedited version of an article accepted for publication in Measurement Science and Technology. IOP Publishing Ltd is not responsible for any errors or omissions in this version of the manuscript or any version derived from it. The definitive publisher authenticated version is available online at

http://dx.doi.org/10.1088/0953-2048/22/11/114014; This is not the published version. Please cite only the published version.; この論文は出版社版でありません。引用の際には出版社版をご確認ご利用ください。 


\title{
Switching current distributions and macroscopic quantum tunneling in over-doped BSCCO mesas with nanometer thickness
}

\author{
I. Kakeya ${ }^{1}$, K. Hamada ${ }^{1}$, T. Tachiki ${ }^{1}$, T. Watanabe ${ }^{2}$, and M. \\ Suzuki ${ }^{1,3}$ \\ ${ }^{1}$ Department of Electronic Science and Engineering, Kyoto University, Nishikyo, \\ Kyoto 615-8510, Japan \\ ${ }^{2}$ Department of Advanced Physics, Hirosaki University, Hirosaki, Aomori 036-8561, \\ Japan \\ ${ }^{3}$ Photonics and Electronics Science and Engineering Center, Kyoto University, \\ Nishikyo, Kyoto 615-8510, Japan \\ E-mail: kakeya@kuee.kyoto-u.ac.jp
}

\begin{abstract}
The current-voltage characteristics and switching dynamics are studied in over-doped $(\mathrm{Pb}, \mathrm{Bi})_{2} \mathrm{Sr}_{2} \mathrm{CaCu}_{2} \mathrm{O}_{8+\delta}(\mathrm{PbBi} 2212)$ and $\mathrm{Bi}_{2} \mathrm{Sr}_{2} \mathrm{Ca}_{2} \mathrm{Cu}_{3} \mathrm{O}_{10+\delta}(\mathrm{Bi} 2223)$ mesa structures containing a few atomic scale intrinsic Josephson junctions (IJJ) along the $c$-axis. The cleave-in-vacuum method enables us to avoid the contact problems that are always accompanied by mesa structures. The highest critical current density of the zero-voltage state as $J_{c 1}=3.2 \mathrm{kA} / \mathrm{cm}^{2}$ among our micron-scale mesa structures of BSCCO cuprates are achieved. Narrowing of the switching probability distribution (SPD) due to suppression of the thermal fluctuation tends to saturate at $5 \mathrm{~K}$, which is regarded as a symptom of macroscopic quantum tunneling (MQT). The crossover temperature between the MQT and the thermal escape regions is estimated as $2.2 \mathrm{~K}$ for the sample with the highest $J_{c 1}$. In the high temperature region, another narrowing in SPD is observed that was attributed to the phase retrapping.
\end{abstract}

PACS numbers: $74.50 .+\mathrm{r}, 74.72 . \mathrm{Hs}, 74.78 . \mathrm{Na}, 85.25 . \mathrm{Cp}$

Submitted to: Supercond. Sci. Technol. 


\section{Introduction}

Intrinsic Josephson junction (IJJ), which is included in high- $T_{c}$ superconductors, provides peculiar properties as a stack of tunnel Josephson junctions (JJs) [1]. Strong coupling between weakly coupled Josephson junctions that is attributed to single-atomic-layer thickness of superconducting electrodes provides a great variety of phenomena $[2,3,4]$, including various Josephson vortex dynamics in $\mathrm{Bi}_{2} \mathrm{Sr}_{2} \mathrm{CaCu}_{2} \mathrm{O}_{8+\delta}$ (Bi2212) single crystals [5, 6] and the emission of coherent terahertz electromagnetic waves [7]. These phenomena are considered collective phenomena among a number of JJs.

Interlayer tunneling spectroscopy (ITS) has been given by a complemental approach through IJJs, where the average of the stacked identical JJs represents a superconductorinsulator-superconductor (SIS) tunnel junction that consists of $\mathrm{CuO}_{2}$ superconducting electrodes and charge reservoir block layers. Since differential conductance $d I / d V$ as a function of biased voltage $V$ in an SIS tunnel junction is a probe of the quasiparticle density of the states in $\mathrm{CuO}_{2}$ layers, ITS, as well as scanning tunneling spectroscopy (STS) and angular resolved photoemission spectroscopy (ARPES), contributes to understanding the mechanism of high- $T_{c}$ superconductivity $[8,9]$. However, the experimental results of ITS include ambiguity about the number of stacked-JJ $N$ because $N$ has not been less than the order of 10 so far. Quite recently, You et al. stimulated the relevance of investigation in samples consist of a single IJJ in order to avoid the ambiguity. Subsequently, we successfully prepared samples with a few IJJ's of mesa and bridge structures[10, 11].

On the other hand, macroscopic quantum tunneling (MQT) in a JJ has been investigated for the phase quantum bit (qubit) implementations since 1980's [12, 13]. The Josephson qubit, which has an advantage for integrated circuit designing despite its short coherence time, has been operated below $100 \mathrm{mK}$, requiring huge cost and time for cooling. Recently, observations of MQT in IJJ close to $1 \mathrm{~K}$ were reported by several groups $[14,15,16,17,18]$. The advantages of IJJ qubit are not only higher operating temperature but also atomic-scale integration due to its crystal structure.

One striking MQT phenomenon attributed to the multiple stacking of IJJs is $N^{2}$ enhancement of the MQT rate $\Gamma_{\mathrm{MQT}}$ reported by Jin et al. [15], who observed that $\Gamma_{\mathrm{MQT}}$ is about $N^{2}$ times larger when the stacked $N$ IJJs simultaneously switch than when only the weakest IJJ among the stacked IJJs is switched. This phenomenon can be explained by considering a long-range coupling between IJJs in which any pairs of IJJs interact, whereas $\Gamma_{\mathrm{MQT}}$ would be enhanced no more than $3 N$ by taking the interaction with the nearest neighbors of IJJs into consideration. Koyama and Machida theoretically argued that the $N^{2}$ enhancement can be driven by the charge coupling between the $\mathrm{CuO}_{2}$ double layers[19], as well as the longitudinal Josephson plasma mode[4, 20]. Stacking effects for escape events including the $N^{2}$ enhancement have not been systematically understood so far. One important reason is that the number of switching junctions remains ambiguous. 
In this paper, we report the fabrication of stacks with a few IJJs from overdoped PbBi2212 and Bi2223 single crystals and the measurements of current-voltage characteristics (IVC) and switching probability distributions (SPD) up to $5 \mathrm{~K}$. The critical current density of the superconductive $(R=0)$ state is more than $1 \mathrm{kA} / \mathrm{cm}^{2}$, which is much higher than the case in Bi2212 and under-doped Bi2223 crystals. The saturation tendencies of the narrowing in the width of SPD were found that imply the crossovers from the TA to the MQT regions.

\section{Theory of phase dynamics in a single Josephson junction}

The current-voltage characteristic (IVC) of a single Josephson junction is described by the RCSJ-model introduced by Stewart [21] and McCumber [22]. This model is formulated as:

$$
\frac{d^{2} \varphi}{d t^{2}}+\frac{1}{Q_{0}} \frac{d \varphi}{d t}+\sin \varphi-\frac{I}{I_{c}}=0
$$

where $\varphi$ is the gauge invariant phase difference between the superconducting electrodes of the Josephson junction, $Q_{0}^{-1}$ is a damping parameter, and $I$ and $I_{c}$ are biased and critical currents, respectively. $Q_{0}=\omega_{p 0} R C$ is given by shunt resistance $R$, shunt capacitance $C$, and Josephson plasma frequency $\omega_{p 0}=\sqrt{2 e I_{c} / \hbar C}$, which represents a quantum phase fluctuation. The phase dynamics described in Eq. (1) is an analogue of a damped motion of a Brownian particle with a mass of $(\hbar / 2 e)^{2} C$ in a tilted cosine (washboard) potential $U(\varphi)=-E_{J}(\cos \varphi+\alpha \varphi)$, where $E_{J}=\hbar I_{c} / 2 e$ is the Josephson coupling energy and $\alpha=I / I_{c}$.

Next, we discuss the underdamped $\left(Q_{0} \gg 1\right)$ case, where $\mathrm{PbBi} 2212$ and $\mathrm{Bi} 2223$ IJJs can be adopted. The zero-voltage (superconducting) state and the resistive state correspond to the particle trapped by barrier $\Delta U$ and running downward along the tilted potential, respectively. Escape from the potential can occur even $\alpha<1$ due to the thermal and the quantum fluctuations (resistive state). When $\alpha$ is decreased from the resistive state, the running particle is not immediately trapped because of its inertia. This results in the large hysteresis in the $I V$ curve of an underdamped JJ. The escape rates by thermal and quantum processes are given by [23]:

$$
\Gamma_{T A}=a_{t} \frac{\omega_{p}}{2 \pi} \exp \left(-\frac{\Delta U}{k_{B} T}\right)
$$

and

$$
\Gamma_{\mathrm{MQT}}=\frac{\omega_{p}}{2 \pi} \sqrt{\frac{864 \Delta U \pi}{\hbar \omega_{p}}} \exp \left(-\frac{36 \Delta U}{5 \hbar \omega_{p}}\right),
$$

respectively. Here, the potential barrier is given by $\Delta U=2 E_{J}\left(\sqrt{1-\alpha^{2}}-\alpha \arccos \alpha\right) \simeq$ $4 \sqrt{2} / 3 E_{J}(1-\alpha)^{\frac{3}{2}}$ with $\alpha \simeq 1, \omega_{p}$ is the current dependent Josephson plasma frequency given by $\omega_{p}=\omega_{p 0}\left(1-\alpha^{2}\right)^{1 / 4}$, and $a_{t}$ is a thermal prefactor that is approximated as 1 in this case. At high temperatures the thermal process is dominant, and the quantum 
process is independent of temperature. With decreasing temperature, $\Gamma_{T A}$ is suppressed and crossovers with $\Gamma_{\mathrm{MQT}}$ at a sufficiently low temperature given by

$$
T_{\mathrm{MQT}} \simeq \frac{\hbar \omega_{p}}{2 \pi k_{B}}
$$

when the quasiparticle dissipation is neglected.

Experimentally, switching probability distribution $P(I)$ from the zero-voltage state to the resistive state is measured as a function of the biased current. Indicating with $N(t)$ the probability that the particle is trapped at time $t$, the probability that the particle escapes from the metastable state during a short interval $d t$ is

$$
d N=-N(t) \tau^{-1} d t=-N(\alpha) \tau^{-1}\left(\frac{d \alpha}{d t}\right)^{-1} d \alpha
$$

where $\tau$ is the lifetime of the metastable state. After integration with initial condition $N(0)=1$, we obtained

$$
P(\alpha)=-\frac{d N}{d \alpha}=-\left(\frac{d \alpha}{d t}\right)^{-1} \tau^{-1}(\alpha) \exp \left[-\int_{0}^{\alpha} \tau^{-1}\left(\alpha^{\prime}\right)\left(\frac{d \alpha^{\prime}}{d t}\right)^{-1} d \alpha^{\prime}\right] .
$$

Considering $\int_{0}^{\alpha} P\left(\alpha^{\prime}\right)=1-N$ and replacing $\alpha$ with $I, P(I)$ is finally given by

$$
P(I)=\left(\frac{d I}{d t}\right)^{-1} \Gamma(I)\left[1-\int_{0}^{I} P(i) d i\right],
$$

where $d I / d t$ is the ramp rate of the bias current and $\Gamma(I)$ is the escape rate of the thermal and the quantum processes.

\section{Experiment}

Single crystals of Bi2223 with a stable composition of $\mathrm{Bi}_{2.1} \mathrm{Sr}_{1.9} \mathrm{Ca}_{2.0} \mathrm{Cu}_{3.0} \mathrm{O}_{10+\delta}$ were grown by the traveling solvent floating (TSFZ) method [24]. To increase critical current $I_{c}$, the crystal was annealed at $800^{\circ} \mathrm{C}$ in flowing oxygen for 100 hours. This oxidization process increased critical temperature $T_{c}$ from 87 to $107 \mathrm{~K}$. Single crystals of PbBi2212 were grown by the self-flux method [25]. Mixed ingredient material with a nominal molar composition of $\frac{1}{2} \mathrm{Bi}_{2} \mathrm{O}_{3}: \mathrm{PbO}: \mathrm{SrCO}_{3}: \mathrm{CaCO}_{3}: \mathrm{CuO}=1.8: 0.2: 2: 1$ : 2 was melted in an $\mathrm{Al}_{2} \mathrm{O}_{3}$ crucible under temperature up to $1000^{\circ} \mathrm{C}$ and slowly cooled to 800 ${ }^{\circ} \mathrm{C}$ at $-1{ }^{\circ} \mathrm{C}$ /hour. A single crystal with flat surfaces (ab-plane) shown in Fig. 1(a) was taken by carefully cleaving a lump of crystals. Electron dispersive spectroscopy (EDS) analysis shows that the distribution of $\mathrm{Pb}$ in the crystal is almost homogenous and the composition is $\mathrm{Bi}_{1.9} \mathrm{~Pb}_{0.1} \mathrm{Sr}_{2.1} \mathrm{Ca}_{0.8} \mathrm{Cu}_{2.0} \mathrm{O}_{8+\delta}$. The $c$-axis lattice constant was found to be $3.09 \mathrm{~nm}$, and the bulk critical temperature $T_{c}$ was measured as $85 \mathrm{~K}$.

Ultra-thin mesa structures were fabricated on the crystal that was glued to a sapphire substrate. To reduce the contact resistance between the electrodes and the topmost IJJs, that always disturbs precise measurement in mesa structures, we deposited a Ag thin film on the fresh surface cleaved inside the vacuum chamber (cleaving-invacuum method) [26], as shown in Fig. 1(b-1), to attain contact resistance as low as $10^{-7}$ 
$\Omega / \mathrm{cm}^{2}$. The details are described elsewhere [10]. The covered surface of the crystal is kept away from deteriorations by vapors and solvents during the subsequent fabrication processes. The mesa structures were formed with electron-beam lithography and Arion milling (Fig. 1(b-2)). The slowest etching rate for Bi2212 is $0.7 \mathrm{~nm} / \mathrm{min}$, which enables control of the thickness layer by layer. Silver electrodes and $\mathrm{SiO}_{2}$ insulation were formed by the lift-off and the self-align methods, respectively (Figs. 1(b-3)(b-4)). Prepared mesas are listed in Table 1

The IVC's were recorded by taking traces on an analog oscilloscope under biasing triangular ac voltage with a constant amplitude to the circuit in which the mesa and a resistor are connected in a series. SPD measurements were done by a time-of-flight method $[13,27]$ with an arbitrary waveform generator and a time interval counter. The applied current waveform consists of a ramp pulse from $I=0$ to current greater than the expected switching current and a starting trigger pulse. This unit including a certain interval is repeated over a period of 12.6 msec. The series resistor is much larger than the case of the IVC measurements so that the measurement circuit is a current-biased Josephson junction. Powder filters that eliminate thermal noise from the environment were installed for the measurements in Bi2223. The sample was cooled with a conventional physical property measurement system (PPMS) by Quantum Design Ltd., so that in the present experiment the lowest temperature is $5 \mathrm{~K}$.

\section{Results and discussions}

\subsection{IV characteristics}

Figure 2 represents IVC in Samples 23A (a) and PbB (b). Clearly there are one superconducting (vertical) $R=0$ and two and five resistive quasiparticle branches with equal separation in the voltage for (a) and (b), respectively. In previous reports in Bi2223 [28] and PbBi2212 [29] mesa structures, the separation between the branches and the maximum current of each branch are less uniform. In Sample 23A, no Bi2212 phase was found in the measurement of the temperature dependence of the $c$ axis resistivity. This is in contrast to the previous result where resistivity drops were found even below $90 \mathrm{~K}$. This achievements are yielded by the cleaving-in-vacuum method and the precise control in the number of stacked IJJs. The return current where the highest resistive branch returns to the superconducting branch in a downward sweep is much smaller than the maximum current of superconducting branch $I_{c 1}$, suggesting the system is a strongly underdamped Josephson junction, $Q_{0} \gg 1$. $I_{c 1}$ is close to the other maximum currents $I_{c 2}, I_{c 3}$, and etc. This is in contrast to the results in pristine Bi2212, where $I_{c 1}$ is greatly suppressed due to a proximity effect of the topmost junction from the surface electrode [18]. Since the quasiparticle penetration is less for a system with higher Cooper pair density, either the post-annealing or the partial substitution of $\mathrm{Bi}$ for $\mathrm{Pb}$ results in higher $I_{c 1}$. The critical current density $J_{c 1}$ is 1.5 and $3.2 \mathrm{kA} / \mathrm{cm}^{2}$ for $23 \mathrm{~A}$ and $\mathrm{PbB}$, respectively, which is larger by more than an order of magnitude than the case in the 
pristine Bi2212.

\subsection{SPD measurements}

Escape rate $\Gamma(I)$ is obtained through experimental $P(I)$ with Eq. (7) as

$$
\Gamma(I)=\frac{1}{\Delta I} \frac{d I}{d t} \ln \frac{\sum_{i \geq I} P(i)}{\sum_{i \geq I-\Delta I} P(i)},
$$

where $\Delta I$ is the step-width of $P(I) . P(I)$ and $\Gamma(I)$ for the 10,000 first switching events in Sample 23A at various bath temperatures $T_{b}$ from 5 to $65 \mathrm{~K}$ are shown in Fig. 3(a). The width of $P(I)$ increases with increasing temperature up to $35 \mathrm{~K}$. Fluctuation-free critical current $I_{c 0}$ and effective temperature $T_{\text {eff }}$ in the single junction TA model are obtained by fitting Eq. (2) to experimental $\Gamma(I)$. The solid curves in the upper panel of Fig. 3 are given by Eq. (3) with the obtained $I_{c 0}$ and $T_{\text {eff }}$ and excellently agree with experimental $P(I)$. Above $35 \mathrm{~K}$, the width of $P(I)$ is decreased with increasing bath temperature $T_{b}$. Although this tendency is not consistent with the TA model, $T_{\text {eff }}$ is evaluated with the same method below $35 \mathrm{~K}$ by using Eq. (2). In this temperature range, slight deviations of the fitted curves from the experimental results are found in the lower tail of $P(I)$. Figure 4 represents the temperature dependence of $T_{\text {eff }}$ for both samples. Three temperature regions are commonly found in $T_{\text {eff }}$ :

(i) Lower temperature region, where $T_{\text {eff }}$ deviates from the TA model and begins to saturate with decreasing $T_{b}$.

(ii) Thermal activation region, where $T_{\text {eff }} \approx T_{b}$.

(iii) Higher temperature region, where $T_{\text {eff }}$ decreases with increasing $T_{b}$.

For Sample 23A, regions (i), (ii), and (iii) correspond to the regions below $10 \mathrm{~K}$, between 10 and $35 \mathrm{~K}$, and above $35 \mathrm{~K}$, respectively. These three regions are common among the results from various groups $[30,18]$. The saturation of $T_{\text {eff }}$ due to MQT is expected at lower temperatures in region (i). Excellent agreements with $T_{\text {eff }}=T_{b}$ in region (ii) strongly suggest that the TA model describes the switching dynamics very well, as discussed by several authors. In region (iii), we anticipate the phase-retrapping process in which thermal activation prevents continuous downward movement of the particle in the tilted washboard potential.

MQT temperatures $T_{\mathrm{MQT}}$ 's were estimated with $I_{c 0}$ at $5.0 \mathrm{~K}$ using Eq. (4), as listed in Table 1. These estimations are supported by previous low temperature experiments in IJJs by many groups. For instance, in our pristine Bi2212 mesas [18], measured crossover temperature $0.4 \mathrm{~K}$ is slightly smaller than estimated $T_{\mathrm{MQT}}=0.57 \mathrm{~K}$. In this work, symptoms were found in region (i), although all $T_{\mathrm{MQT}}$ 's are out of our temperature window. For Sample $\mathrm{PbB}$, the extrapolation of $T_{\text {eff }}$ to lower temperatures implies saturation above $1 \mathrm{~K}$.

To test how close our lowest temperature $5 \mathrm{~K}$ is to the quantum region, MQT rate $\Gamma_{\mathrm{MQT}}$ is estimated by using Eq. (3). Figure 5 compares experimental $\Gamma(I)$ with 
estimated $\Gamma_{\mathrm{MQT}}$ with $I_{c 0}$ values at $5 \mathrm{~K} . \Gamma(I)$ is larger than $\Gamma_{\mathrm{MQT}}$ by many orders of magnitude for all samples. Although it is probably relevant that the measured temperature is not sufficiently low, we would like to discuss three other plausible reasons for these high escape rates.

The first is the multiple switching effect. Since $I_{c 1}$ of $\mathrm{PbB}$ is so close to the maximum currents of the other branches, we did not resolve each switching event between the six branches; $P(I)$ includes not only single-junction switching events but also multi-junction switching events. As reported by Jin et al., $\Gamma(I)$ for multi-junction switching events in the stack with $N$ IJJs is enhanced by the order of $N^{2}$. It is impossible to evaluate the enhancement because we do not reach to sufficiently low temperatures, where $T_{\text {eff }}$ is certainly independent of $T_{b}$. On the other hand, the difference between $I_{c 1}$ and $I_{c 2}$ in $23 \mathrm{~A}$ is sufficient to resolve the first and the second switchings. Thus $P(I)$ shown in Fig. 3(a) does not include the multiple-junction switching. This might explain why deviation from the TA model is smaller in 23A than in $\mathrm{PbB}$.

The second is the large junction effect. When the junction size is larger than the Josephson penetration depth $\lambda_{J}$, i.e., phase difference $\varphi$ spatially varies, the escape rate is enhanced. This phenomena can be understood by considering a one-dimensional array of particles in the two-dimensional washboard potential [31, 32]. When one particle starts to escape from the barrier, the neighboring particles follow the escaping particle. The Josephson penetration depth for an IJJ is given by $\lambda_{J} \equiv \sqrt{\hbar t / 4 e \mu_{0} J_{c} \lambda^{2}}$ with $\lambda=\lambda_{a b} t /(t+d)$, where $t, d$, and $\lambda_{a b}$ are the thickness of the superconducting atomic layer $(0.3 \mathrm{~nm}$ for Bi2212 and $0.65 \mathrm{~nm}$ for Bi2223), the thickness of the block layers (1.2 nm for both materials), and the $a b$-plane penetration depth, respectively [33]. Using respective $J_{c}=I_{c 0} / A$ and $\lambda_{a b}=170 \mathrm{~nm}$ for both materials, $\lambda_{J}$ 's for Samples $23 \mathrm{~A}$ and $\mathrm{PbB}$ are estimated as 1.23 and $0.76 \mu \mathrm{m}$, respectively, which are rather smaller than the junction lengths. Therefore we have to consider the large junction effect.

The other is noise from the environments. It is easy to imagine that the fluctuation of the bias current attributed noise which penetrated the signal lines, and gives extrinsic fluctuation of the switching events, resulting in an increase in $\Gamma(I)$. The signal lines of the MQT measurement setup often include low-pass powder filters whose cut-off frequencies are of the order of $1 \mathrm{GHz}$. In this work, a set of powder filters was used for measurements in 23A. This may be one reason that $T_{\text {eff }}$ deviates at higher temperatures in $\mathrm{PbB}$ than in $23 \mathrm{~A}$, even if both $T_{\text {eff }}$ and $T_{b}$ are normalized by $T_{\mathrm{MQT}}$.

In the present experiment, the heating of the mesas, electrodes, and their interfaces is almost negligible. According to our numerical simulation based on the Fourier's low, temperature rise $\Delta T$ of a $2 \mu \mathrm{m}$ square, $15 \mathrm{~nm}$ thick mesa is $1.8 \mathrm{~K}$ at an injection current density of $2 \times 10^{4} \mathrm{~A} / \mathrm{cm}^{2}$ at a base temperature of $10 \mathrm{~K}$ [34]. In the present case, since the current density is $\sim 2 \times 10^{3} \mathrm{~A} / \mathrm{cm}^{2}$, we expect $\Delta T \sim 0.2 \mathrm{~K}$ because the Joule heating is proportional to the product of the square of the current density $(\times 1 / 100)$ and the conductance $(\times 10)$. By using other methods from previous papers on SPD in IJJs, $\Delta T$ is estimated to be not more than $0.2 \mathrm{~K}$. Thus the heating should be considered a minor effect for large $T_{\text {eff }}$. 
The collapse of $T_{\text {eff }}$ in region (iii) is attributed to phase-retrapping by thermal fluctuation [30], in which a particle that escapes from a barrier is trapped by another barrier. When retrapping rate is larger than thermal escape rate $\Gamma_{T A}$, the probability of switching events is suppressed. In this case, the suppression of the escape rate at lower currents is more pronounced, resulting that skewness of $P(I)$ changes in this temperature region as clearly simulated by Fenton and Warburton [35]. As seen in Fig. 3, suppression of $\Gamma(I)$ at lower current is remarkable below and above the onset of the collapse $T^{*}$, and that at higher current monotonically increases with increasing $T_{b}$. This result is consistent with the picture of phase-retrapping. $T^{*}$ is much higher than the case in Ref. [18]. This difference is easily explained by considering the difference in Josephson coupling energy $E_{J}$. Larger thermal fluctuation is required to trap an escaped point from a higher potential barrier by assuming the same damping parameter $Q_{0}$. Krasnov treats this phenomenon systematically among junctions with various $Q_{0}$ 's and indicates the relation between $k_{B} T^{*} / E_{J}$ and $Q_{0}$ in Fig. 20 of Ref. [30]. The sets of $\left(Q_{0}, k_{B} T^{*} / E_{J}\right)$ for Samples $23 \mathrm{~A}$ and $\mathrm{PbB}$ are estimated as $(129,0.025)$ and $(94,0.042)$, respectively. These values roughly agree with their extrapolations within an order of magnitude, although the tendency among our two values is opposite. This is partly due to the ambiguity in $R$ for estimating $Q_{0}$. Further systematic investigations on IJJs with higher $Q_{0}$ might reveal the whole features of the retrapping phenomena.

\section{Summary}

We investigated IVC and the switching dynamics of PbBi2212 and Bi2223 IJJ mesa structures. Because of higher carrier concentrations, the suppression of $J_{c}$ of the topmost IJJ is reduced in contrast to the case in pristine Bi2212, which has been previously reported. In a PbBi2212 mesa, fluctuation free critical current $I_{c 0}$ estimated from the $\mathrm{SPD}$ at $5 \mathrm{~K}$ yields MQT crossover temperature $T_{\mathrm{MQT}}$ as $2.2 \mathrm{~K}$, which is the highest value among BSCCO families previously reported. Precise control of the number of stacked atomic Josephson junctions enables us to investigate MQT and TA escape rates qualitatively as a function of the number of switching IJJs. Although practical experiments and discussions remain for coming issues, this approach definitely helps us understand the coup ling mechanisms of IJJs.

\section{Acknowledgments}

This work was supported by Global COE project "Education and Research on Photonics and Electronics Science and Engineering" at Kyoto University and the Core-to-Core Program "Nanoscience and Engineering in Superconductivity" by Japan Society for the Promotion of Science (JSPS).

[1] R. Kleiner and P. Müller, "Intrinsic Josephson effects in high- $T_{c}$ superconductors," Phys. Rev. B, vol. 49, pp. 1327-1341, Jan 1994.

[2] S. Sakai, P. Bodin, and N. F. Pedersen, "Fluxons in thin-film superconductor-insulator superlattices," J. Appl. Phys., vol. 73, p. 2411, 1993. 
[3] T. Koyama and M. Tachiki, " $I-V$ characteristics of Josephson-coupled layered superconductors with longitudinal plasma excitations," Phys. Rev. B, vol. 54, p. 16183, 1996.

[4] M. Machida, T. Koyama, and M. Tachiki, "Dynamical breaking of charge neutrality in intrinsic Josephson junctions: common origin for microwave resonant absorption and multiple-branch structures in the $I-V$ characteristics," Phys. Rev. Lett., vol. 83, p. 4618, 1999.

[5] I. Kakeya, T. Wada, R. Nakamura, and K. Kadowaki, "Two Phase Collective Modes in a Josephson Vortex Lattice in the Intrinsic Josephson Junction $\mathrm{Bi}_{2} \mathrm{Sr}_{2} \mathrm{CaCu}_{2} \mathrm{O}_{8+\delta}$," Phys. Rev. B, vol. 72, pp. 014540-1-10, 2005.

[6] I. Kakeya, Y. Kubo, M. Kohri, M. Iwase, T. Yamamoto, and K. Kadowaki, "Scaling behavior of the crossover to short-stack regimes of Josephson vortex lattices in $\mathrm{Bi}_{2} \mathrm{Sr}_{2} \mathrm{CaCu}_{2} \mathrm{O}_{8+\delta}$ stacks," Phys. Rev. B, vol. 79, no. 21, p. 212503, 2009.

[7] L. Ozyuzer, A. Koshelev, C. Kurter, N. Gopalsami, Q. Li, M. Tachiki, K. Kadowaki, T. Yamamoto, H. Minami, H. Yamaguchi, T. Tachiki, K. Gray, W. Kwok, and U. Welp, "Emission of coherent THz radiation from superconductors," SCIENCE, vol. 318, no. 5854, pp. 1291 - 1293, 2007.

[8] M. Suzuki, T. Watamnabe, and A. Matsuda, "Interlayer tunneling spectroscopy for slightly overdoped $\mathrm{Bi}_{2} \mathrm{Sr}_{2} \mathrm{CaCu}_{2} \mathrm{O}_{8+\delta}$," Phys. Rev. Lett., vol. 82, p. 5361, 1999.

[9] V. Krasnov, A. Yurgens, D. Winkler, P. Delsing, and T. Claeson, "Evidence for coexistence of the superconducting gap and the pseudogap in Bi-2212 from intrinsic tunneling spectroscopy," Phys. Rev. Lett., vol. 84, pp. 5860-5863, JUN 192000.

[10] R. Takemura, M. Ohmaki, T. Kumano, T. Watanabe, and M. Suzuki, "Fabrication of small mesa structures of $\mathrm{Bi}_{2} \mathrm{Sr}_{2} \mathrm{CaCu}_{2} \mathrm{O}_{8+\delta}$ intrinsic josephson junctions using polyimide process: Improvement of current-voltage characteristics with a very small number of junctions," Jap. J. Appl. Phys., vol. 46, pp. 5788-5791, SEP 2007.

[11] A. Yurgens, M. Torstensson, L. X. You, T. Bauch, D. Winkler, I. Kakeya, and K. Kadowaki, "Small-number arrays of intrinsic josephson junctions," Physica C, vol. 468, pp. 674-678, APR 12008.

[12] R. Voss and R. Webb, "Macroscopic Quantum Tunneling In $1 \mu \mathrm{m}$ Nb Josephson-Junctions," Phys. Rev. Lett., vol. 47, no. 4, pp. 265-268, 1981.

[13] J. Martinis, M. Devoret, and J. Clarke, "Experimental tests for the quantum behavior of a macroscopic degree of freedom - The phase difference across a Josephson junction," Phys. Rev. $B$, vol. 35, pp. 4682-4698, APR 11987.

[14] K. Inomata, S. Sato, K. Nakajima, A. Tanaka, Y. Takano, H. Wang, M. Nagao, H. Hatano, and S. Kawabata, "Macroscopic quantum tunneling in a $d$-wave High- $T_{c} \mathrm{Bi}_{2} \mathrm{Sr}_{2} \mathrm{CaCu}_{2} \mathrm{O}_{8+\delta}$ superconductor," Phys. Rev. Lett., vol. 95, no. 10, p. 107005, 2005.

[15] X. Y. Jin, J. Lisenfeld, Y. Koval, A. Lukashenko, A. V. Ustinov, and P. Müller, "Enhanced Macroscopic Quantum Tunneling in $\mathrm{Bi}_{2} \mathrm{Sr}_{2} \mathrm{CaCu}_{2} \mathrm{O}_{8+\delta}$ Intrinsic Josephson-Junction Stacks," Phys. Rev. Lett., vol. 96, no. 17, p. 177003, 2006.

[16] S.-X. Li, W. Qiu, S. Han, Y. F. Wei, X. B. Zhu, C. Z. Gu, S. P. Zhao, and H. B. Wang, "Observation of Macroscopic Quantum Tunneling in a Single $\mathrm{Bi}_{2} \mathrm{Sr}_{2} \mathrm{CaCu}_{2} \mathrm{O}_{8+\delta}$ Surface Intrinsic Josephson Junction," Phys. Rev. Lett., vol. 99, no. 3, p. 037002, 2007.

[17] H. Kashiwaya, T. Matsumoto, H. Shibata, S. Kashiwaya, H. Eisaki, Y. Yoshida, S. Kawabata, and Y. Tanaka, "Switching Dynamics of Bi2Sr2CaCu2O8+delta Intrinsic Josephson Junctions: Macroscopic Quantum Tunneling and Self-Heating Effect," J. Phys. Soc. Jpn., vol. 77, p. 104708, OCT 2008.

[18] K. Ota, K. Hamada, R. Takemura, M. Ohmaki, T. Machi, K. Tanabe, M. Suzuki, A. Maeda, and H. Kitano, "Comparative study of macroscopic quantum tunneling in $\mathrm{Bi}_{2} \mathrm{Sr}_{2} \mathrm{CaCu}_{2} \mathrm{O}_{y}$ intrinsic Josephson junctions with different device structures," Phys. Rev. B, vol. 79, p. 1234505, APR 2009 .

[19] M. Machida and T. Koyama, "Collective dynamics of macroscopic quantum tunnelling in layered high- $T_{c}$ superconductors," Supercond. Sci. Technol., vol. 20, pp. S23-S27, FEB 2007.

[20] I. Kakeya, K. Kindo, K. Kadowaki, S. Takahashi, and T. Mochiku, "Mode Separation of the 
Table 1. List of samples. $T_{c}, J c 1, A, N$, and $T_{\mathrm{MQT}}$ represent critical temperature, maximum current of the superconducting branch at $5 \mathrm{~K}$, area of the junction, number of IJJs, and expected crossover temperature, respectively.

\begin{tabular}{ccccccc}
\hline Name & Material & $T_{c}[\mathrm{~K}]$ & $J_{c 1}\left[\mathrm{~A} / \mathrm{cm}^{2}\right]$ & $A\left[\mu \mathrm{m}^{2}\right]$ & $N$ & $T_{\mathrm{MQT}}$ \\
\hline $23 \mathrm{~A}$ & $\mathrm{Bi} 2223$ & 107 & 1500 & $2 \times 2$ & 2 & 1.4 \\
$\mathrm{PbA}$ & $\mathrm{PbBi} 2212$ & 86 & 1700 & $2 \times 2$ & 2 & 1.8 \\
$\mathrm{PbB}$ & $\mathrm{PbBi} 2212$ & 86 & 2300 & $1 \times 1$ & 5 & 2.2 \\
\hline
\end{tabular}

Josephson Plasma in $\mathrm{Bi}_{2} \mathrm{Sr}_{2} \mathrm{CaCu}_{2} \mathrm{O}_{8+\delta}$," Phys. Rev. B, vol. 57, pp. 3108-3115, 1998.

[21] W. C. Stewart, "Current-voltage characteristics of josephson junctions," Appl. Phys. Lett., vol. 12, no. 8, pp. 277-\&, 1968.

[22] D. E. McCumber, "Effect of ac impedance on dc voltage-current characteristics of superconductor weak-link junctions," J. Appl. Phys., vol. 39, no. 7, pp. 3113-\&, 1968.

[23] T. A. Fulton and L. N. Dunkleberger, "Lifetime of zero-voltage state in Josephson tunneljunctions," Phys. Rev. B, vol. 9, no. 11, pp. 4760-4768, 1974.

[24] T. Fujii, T. Watanabe, and A. Matsuda, "Single-crystal growth of $\mathrm{Bi}_{2} \mathrm{Sr}_{2} \mathrm{Ca}_{2} \mathrm{Cu}_{3} \mathrm{O}_{10+\delta}(\mathrm{Bi}-2223)$ by TSFZ method," J. of Crystal Growth, vol. 223, pp. 175-180, FEB 2001.

[25] A. Irie, M. Sakakibara, and G. Oya, "Growth and tunneling properties of $(\mathrm{Bi}, \mathrm{Pb})_{2} \mathrm{Sr}_{2} \mathrm{CaCu}_{2} \mathrm{O}_{y}$ single-crystals," IEICE Transactions on Electronics, vol. E77C, pp. 1191-1198, AUG 1994.

[26] S. P. Zhao, X. B. Zhu, Y. F. Wei, G. H. Chen, Q. S. Yang, and C. T. Lin, " $\mathrm{Bi}_{2} \mathrm{Sr}_{2} \mathrm{CaCu}_{2} \mathrm{O}_{8+\delta}$ intrinsic Josephson junctions: Surface layer characterization and control," Phys. Rev. B, vol. 72, p. 184511, NOV 2005.

[27] A. Wallraff, A. Lukashenko, C. Coqui, A. Kemp, T. Duty, and A. Ustinov, "Switching current measurements of large area Josephson tunnel junctions," Rev. Sci. Instr., vol. 74, pp. 37403748, AUG 2003.

[28] Y. Yamada, K. Anagawa, T. Shibauchi, T. Fujii, T. Watanabe, A. Matsuda, and M. Suzuki, "Interlayer tunneling spectroscopy and doping-dependent energy-gap structure of the trilayer superconductor $\mathrm{Bi}_{2} \mathrm{Sr}_{2} \mathrm{Ca}_{2} \mathrm{Cu}_{3} \mathrm{O}_{10+\delta}$," Phys. Rev. B, vol. 68, AUG 12003.

[29] A. Irie, T. Mimura, M. Okano, and G. Oya, "Intrinsic Josephson tunnelling properties in smallsized Bi-Pb-Sr-Ca-Cu-Cu-O mesas," Supercond. Sci. Technol., vol. 14, pp. 1097-1101, DEC 2001.

[30] V. M. Krasnov, T. Golod, T. Bauch, and P. Delsing, "Anticorrelation between temperature and fluctuations of the switching current in moderately damped Josephson junctions," Phys. Rev. B, vol. 76, p. 224517, DEC 2007.

[31] M. Castellano, G. Torrioli, C. Cosmelli, A. Costantini, F. Chiarello, P. Carelli, G. Rotoli, M. Cirillo, and R. Kautz, "Thermally activated escape from the zero-voltage state in long Josephson junctions," Phys. Rev. B, vol. 54, pp. 15417-15428, DEC 11996.

[32] T. Kato and M. Imada, "Macroscopic quantum tunneling of a fluxon in a long Josephson junction," J. Phys. Soc. Jpn., vol. 65, pp. 2963-2975, SEP 1996.

[33] T. Kawakami and M. Suzuki, "Direct observation of intrinsic Josephson junction characteristics in electron-doped $\mathrm{Sm}_{2-x} \mathrm{Ce}_{x} \mathrm{CuO}_{4-\delta}$," Phys. Rev. B, vol. 76, p. 134503, OCT 2007.

[34] M. Suzuki, Y. Yamada, E. Tajitsu, and S. Kojima, "Self-heating in small mesa structures made of intrinsic Josephson junctions in BSCCO," IEEE Transactions on Applied Superconductivity, vol. 17, pp. 594-597, JUN 2007.

[35] J. C. Fenton and P. A. Warburton, "Monte Carlo simulations of thermal fluctuations in moderately damped Josephson junctions: Multiple escape and retrapping, switching- and return-current distributions, and hysteresis," Phys. Rev. B, vol. 78, p. 054526, AUG 2008. 

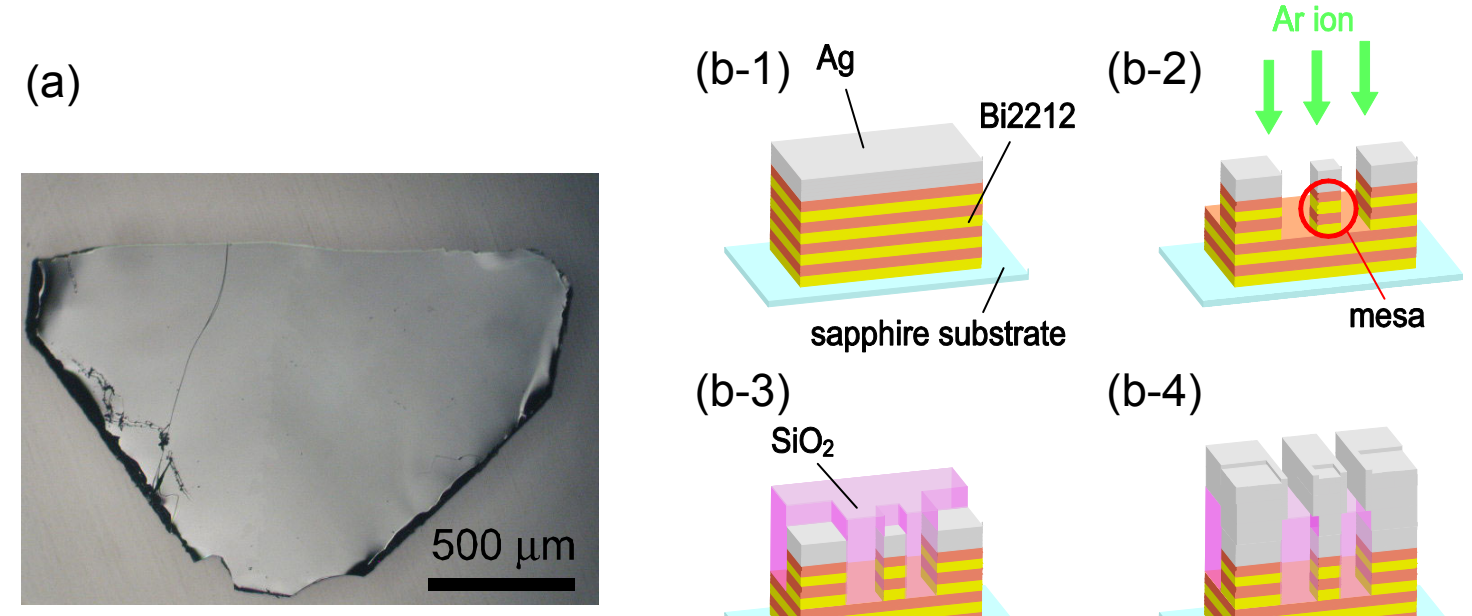

(b-3)

(b-4)
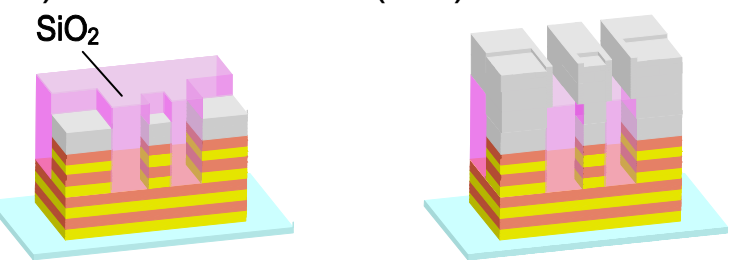

Figure 1. Prepared PbBi2212 single crystal (a) and process of fabrication (b). See text.
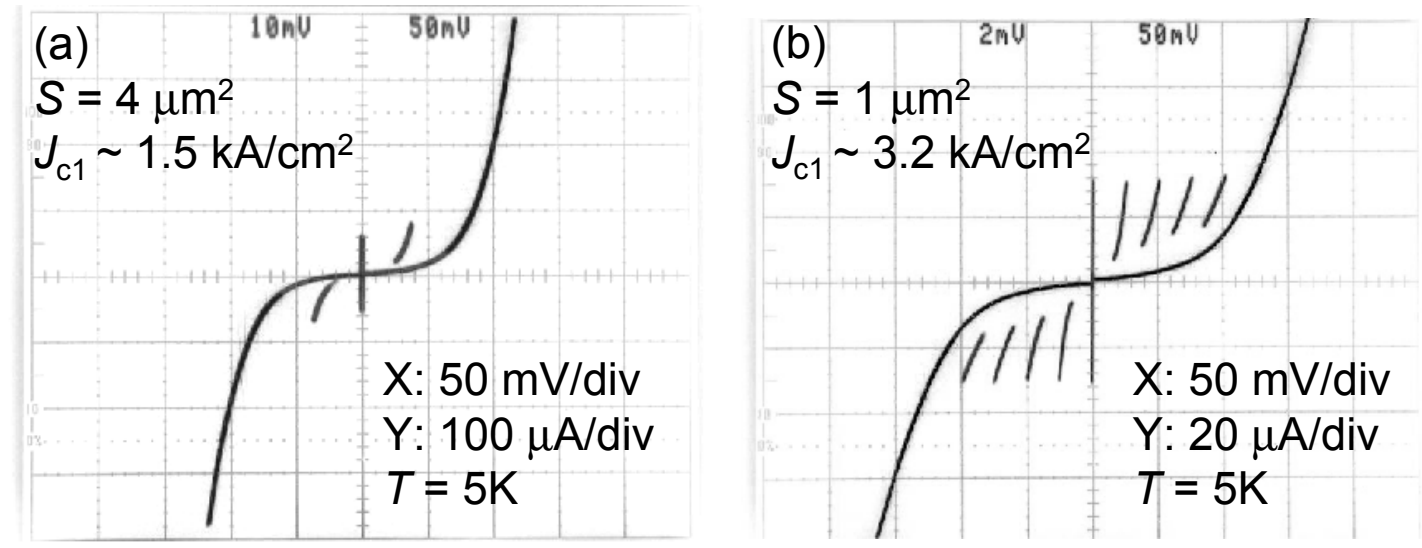

Figure 2. IVC in 23A (a) and $\mathrm{PbB}(\mathrm{b})$. Vertical and horizontal axes correspond with current and voltage axes, respectively. Grid scales are indicated in the panels. 

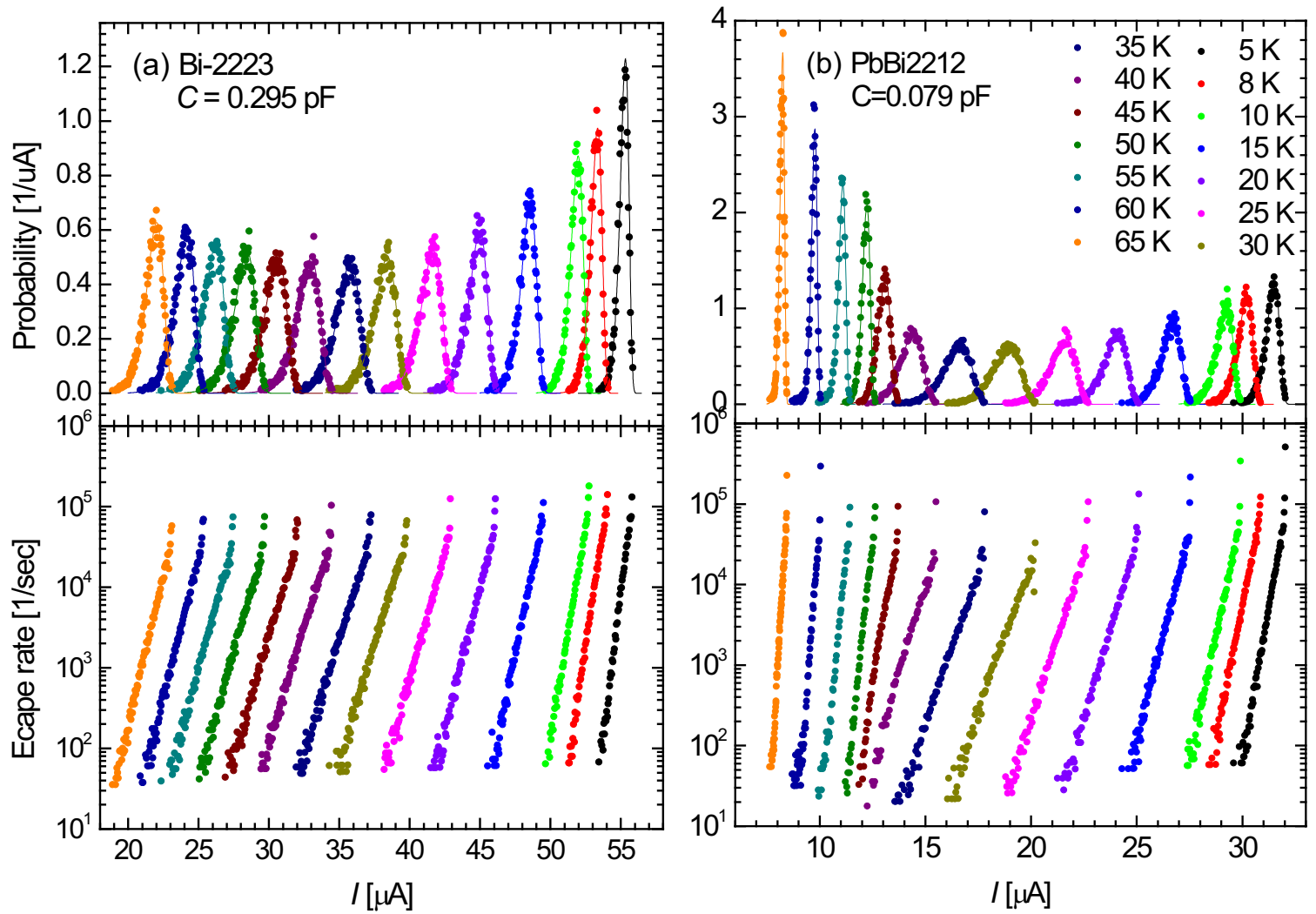

Figure 3. Switching current distribution $P(I)$ and escape rate $\Gamma(I)$ in $23 \mathrm{~A}$ (a) and $\mathrm{PbB}$ (b). Temperatures are 5, 8, 10, 15, 20, 25, 30, 35, 40, 45, 50, 55, 60, and $65 \mathrm{~K}$ from right to left for (a) and (b). Solid curves in upper panels are calculated results of Eq. (7) with parameters obtained by fitting Eq. (2) to the data in the lower panels. 


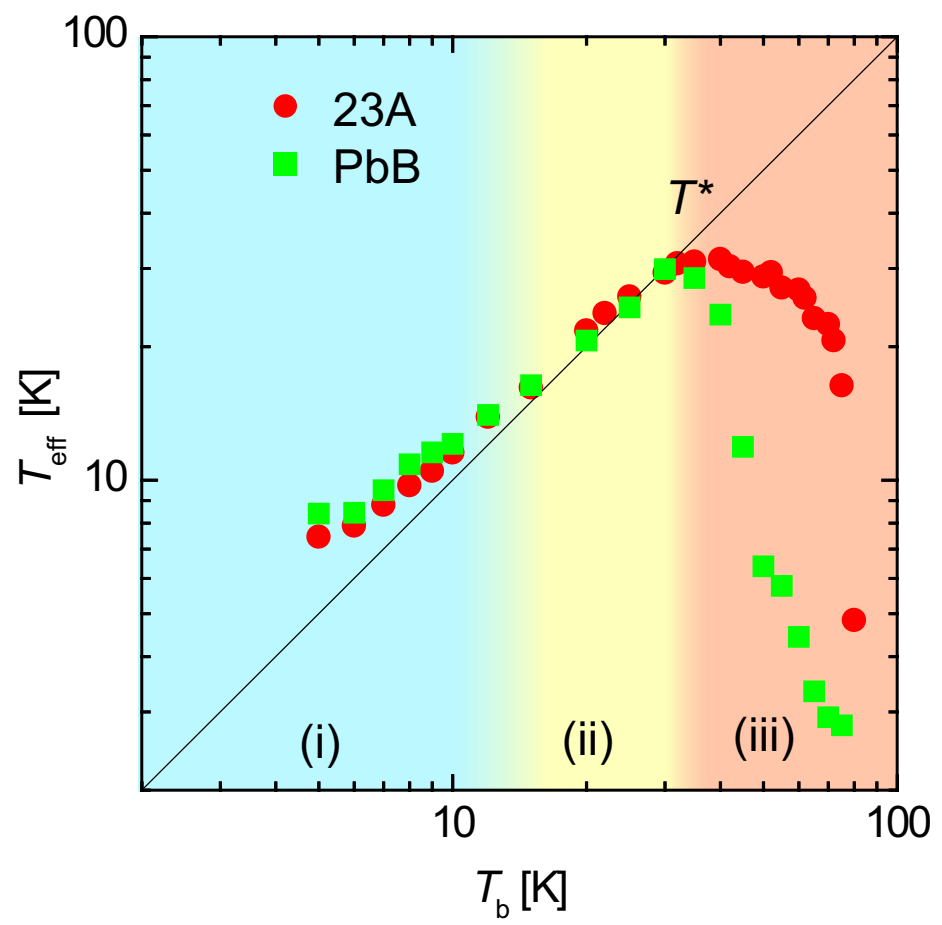

Figure 4. Effective temperatures obtained by fitting Eq. (2) to the measured escape rate $\Gamma(I)$ as a function of bath temperature $T_{b}$. The background is colored for three temperature regions mentioned in the text.

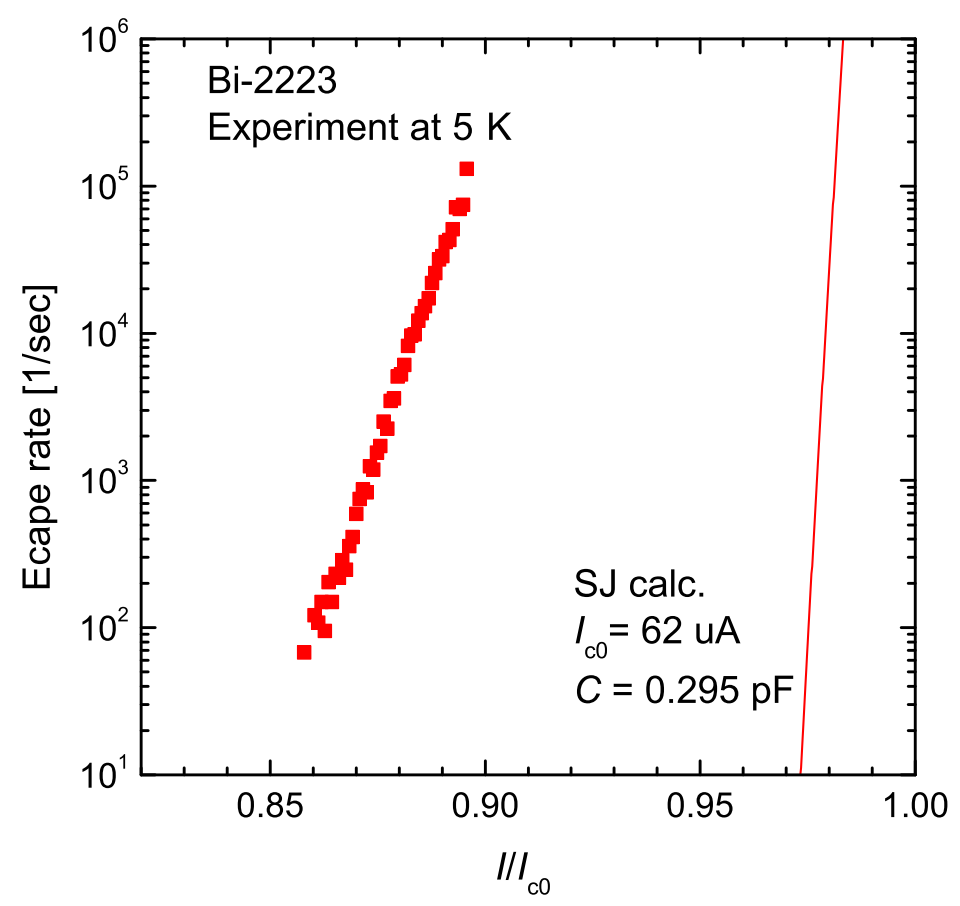

Figure 5. Comparison between $\Gamma(I)$ at $5 \mathrm{~K}$ and expected MQT rate calculated by Eq. (3) with parameters obtained from SPD measurements at $5 \mathrm{~K}$. 